\title{
Mathematical Models of Evolution for Replicator Systems: Fitness Landscape Adaptation
}

\author{
Bratus A.S. ${ }^{1,2}$, Yakushkina T. ${ }^{3}$, Drozhzhin S. ${ }^{1}$, Samokhin I. ${ }^{1}$, \\ ${ }^{1}$ Faculty of Computational Mathematics and Cybernetics, Lomonosov Moscow State University, \\ Moscow, Russia \\ ${ }^{2}$ Department of Applied Mathematics 1, Russian University of Transport, Moscow, Russia \\ ${ }^{3}$ School of Business Informatics, National Research University Higher School of Economics, \\ Moscow, Russia \\ alexander.bratus@yandex.ru
}

Two classical approaches to replicator systems are considered: quasispecies and hypercycle models. We expand the adaptive landscape metaphor by S. Wright and Fisher's fundamental theorem of natural selection, combining it with Kimura's maximal principals to the case of dynamical fitness landscape. We assume that the parameters of the replicator system depend continuously on the evolutionary time, which distinguish from the internal system dynamics time. We suppose that evolutionary time is much slower that ordinary time of the system. Each step of the process of the fitness landscape adaptation place occurs in a steady-state. This process is equivalent to maximization the mean fitness of the system. From mathematical point of view, it is reduced to a series mathematical programming problems or to a first eigenvalue maximization problem. New properties of the adapted systems are discussed.

Key words: model of evolution, hypercycle system, fitness landscape, error threshold, quasispecies model, optimization process.

\section{Математические модели эволюции репликаторных систем: адаптация ландшафта}

\section{приспособленности}

\author{
Братусь А.С. ${ }^{1,2}$, Якушкина Т.С. ${ }^{3}$, Дрожжин С.В. ${ }^{1}$, Самохин И.А. ${ }^{1}$ \\ ${ }^{1}$ Факультет вычислительной математики и кибернетики, Московский государственный \\ университет им. М.В. Ломоносова, Москва, Россия \\ ${ }^{2}$ Кафедра прикладной математики - 1, Российский университет транспорта (МИИТ), \\ Москва, Россия \\ ${ }^{3}$ Школа бизнес-информатики, Нациионльньй исследовательский университет \\ «Высшая школа экономики», Москва, Россия
}

В работе рассматриваются два классических случая формализации репликаторных систем: модели квазивидов и гиперциклической репликации. Основываясь на концепциях Райта и Фишера об адаптивном ландшафте приспособленности и фундаментальной теореме естественного отбора, дополняя их принципом максимума работ Кимуры, мы предлагаем метод анализа систем с динамическим ландшафтом приспособленности. В исследовании предполагается, что параметры репликаторной системы непрерывно зависят от эволюционного времени, которое предлагается отличать от времени внутренней динамики системы. Кроме того, считаем, что адаптивные изменения ландшафта приспособленности происходят в положении равновесия системы, поскольку эволюционное время адаптации намного медленнее времени внутренней динамики. Весь процесс представлен как максимизация средней приспособленности популяции в системе. С точки зрения математической формализации, задача сводится либо к серии задач математического 
программирования, либо к максимизации главного собственного значения. Обсуждается ряд новых результатов, описывающих свойства адаптированной системы.

Ключевые слова: модели эволюции, система гиперииклической репликации, ландиафт приспособленности, порог ошибок, модель квазивидов, оптимизационньй процесс.

\section{Introduction}

\subsection{Replicator systems}

One of the approaches to describe evolutionary processes is replicator systems modeling. This class of models includes such formalizations as replicator equations, quasispecies systems, adaptive dynamics, and Price equations [1]. These models suggest different ways to describe fundamental evolutionary forces: selection and mutation. In many cases, this means using the model with a particular constant fitness landscape, when any genotype or phenotype corresponds to its fitness value. However, for practical applications, it is reasonable to consider the impact of changing environment and, thus, dynamical fitness landscapes. There are several ways to include these variations in the fitness landscape, e.g., considering random evolutionary parameters [2,3] or some optimization process over these parameters $[4,5]$

In this paper, we discuss the underlying concept of the recent studies $[4,5]$, which propose a new method of fitness landscape adaptation and describe it in the form of the optimization problem. One of the first researchers, who suggested using the extreme principle for Darwinian evolution, was R. Fisher. A famous theorem states: "The rate of increase in (mean) fitness of any organism at any time is equal to its genetic variance in fitness at that time" [6]. Following this idea, together with the adaptive fitness landscape concept [7], we expand it on the case of the dynamical evolutionary parameters that respond itself to the systems dynamics and environment.

\section{Fitness landscape adaptation process in hypercycle systems}

\subsection{Hypercycle systems}

One of specific cases of replicator equations are hypercycle systems, which were introduced in the seminal studies by Eigen and Schuster [8, 9] for the prebiotic evolution hypothesis. This concept could explain the beginning of life suggesting the existence of a universal macromolecule - predecessor of the RNA and DNA molecules. In more general sense, it is a technique that allows modelling chemical reactions in hypercycles: the evolution of macromolecules, which facilitate catalyzis of their neighboughs. The hypercycle system is a useful model self-organizing object with interesting mathematical properties: hypercycles are permanent; hypercycle systems with an order higher than 5 has trajectories converging to a stable limit cycle; hyper cycle systems can change over time if new molecules are included.

The central hypothesis of this study is that the specific time of the evolutionary adaptation of the system parameters is much slower than the time of the internal evolutionary process. We call the first one evolutionary time. This assumption leads to the fact that evolutionary changes of the system parameters happen in a steady-state of the corresponding dynamical system. Hence, the problem statement can be interpreted as follows: the maximization of the mean fitness in a steady-state is performed by varying the system parameters under the condition of bounded values of these parameters.

\subsection{Problem Statement}

The first class of the systems considered - the hyper cycle systems - is a variant of the classical replicator equations:

$$
\dot{u}_{i}=u_{i}\left((\mathbf{A u})_{i}-\bar{m}(\mathbf{u})\right), \quad i=1, \ldots n .
$$

Here, $\mathbf{A}=\left[a_{i j}\right]$ is a given $n \times n$ matrix of fitness coefficients. Value $(\mathbf{A u})_{i}$ is $i$-th element of the vector $\mathbf{A u}$, where vector $\mathbf{u}$ stands for the distribution of the species in the population over time.

$$
\begin{gathered}
\mathbf{u}(\mathrm{t}) \in \mathrm{S}_{\mathrm{n}}=\left\{\mathrm{x} \in \mathrm{R}^{\mathrm{n}}: \mathrm{x} \geq 0, \sum_{i=1}^{n} x_{i}=1\right\}, \\
\bar{m}(\mathbf{u})=\sum_{i, j=1}^{n} a_{i j} u_{i} u_{j}=\langle\mathbf{A u}, \mathbf{u}\rangle .
\end{gathered}
$$

We assume that the elements of matrix $\mathbf{A}(\tau)$ depend continuously on the evolutionary time $\tau \in[0, \infty)$, which is much slower than system time $t$. This implies, in terms of numerical calculations, that after each step of the parameters adaptation process the system has enough time to find itself either in a stationary state $\hat{\mathbf{u}}$ if it exists and is globally stable, or at some other asymptotic regime:

$$
\mathbf{A}(\tau) \hat{\mathbf{u}}=\bar{m}(\hat{\mathbf{u}}) \mathbf{1}, \mathbf{1}=(1,1, \ldots, 1), \hat{\mathbf{u}} \in S_{n} .
$$

One possible approach to include restriction on the resourcesis to assume that the sum of the squares of the elements of $\mathbf{A}(\tau)$ have limited growths during the whole evolutionary process by some constant $Q$ :

$$
\sum_{i, j=1}^{n} a_{i j}^{2}(\tau) \leq Q^{2}, \tau \geq 0 .
$$

To rephrase the problem in terms of the described system, we consider the following optimization problem: to find such elements $a_{i j}$ of matrix $\mathbf{A}(\tau)$ satisfying (4) such that the mean integral fitness $\bar{m}(\tau)$ of the population, defined by (3), is maximized at some fixed time moment $\tau=T$ of the evolutionary time. 


\subsection{Optimization process}

We divide the interval $\tau \in[0, T]$ into smaller steps of the magnitude $h$ and expand the values $\mathbf{A}, \bar{m}, \hat{\mathbf{u}}$, according to the following scheme:

$$
\mathbf{A}(\tau+h)=\mathbf{A}_{0}(\tau)+\mathbf{A}_{1}(\tau) h+o(h) .
$$

Then, we derive the expression for the fitness variation (linear part):

$$
m_{1}=m_{0}^{2}\left\langle\mathbf{A}_{0}^{-1} \mathbf{A}_{1} \mathbf{A}_{0}^{-1} \mathbf{1}, \mathbf{1}\right\rangle=m_{0}\left\langle\mathbf{A}_{0}^{-1} \mathbf{A}_{1} \mathbf{u}_{0}, \mathbf{1}\right\rangle .
$$

Moreover, the constraints (4) lead to:

$$
\begin{aligned}
& \sum_{i, j=1}^{n} a_{i j}(\tau) a_{i j}^{\prime}(\tau) \leq 0, \\
& \left|a_{i j}^{\prime}\left(\tau_{0}\right)\right| \leq \varepsilon, \quad \varepsilon>0 .
\end{aligned}
$$

Hence, the optimization problems of the fitness maximization on the interval $\Delta \tau$ turns into a linear programming problem to maximize fitness variation (6) subject to the constraints (4), (7) and (8).

Updating the values $\mathbf{A}(\tau+h), \bar{m}(\tau+h)$ and calculating a new equilibrium $\hat{\mathbf{u}}(t+h)$ at each step, we reduce the maximization problem to a sequence of linear programming problems (detailed analysis is presented in [4]).

\subsection{Example and discussion}

Consider an example of the hypercycle system with five macromolecules and a parasite.

$$
\begin{gathered}
\dot{u}_{i}=u_{i}\left(u_{i-1}-f(\mathbf{u})\right), \quad i=1,2 \ldots 5, \\
\dot{u}_{6}=u_{6}\left(1.7 u_{5}-f(\mathbf{u})\right), \\
f(\mathbf{u})=\sum_{i=1}^{5} u_{i} u_{i-1}+1.7 u_{5} u_{6}, \quad \sum_{i=1}^{6} u_{i}=1, \quad u_{1}=u_{5} .
\end{gathered}
$$

In [4], we showed the following properties of this system class:

- Evolutionary process of the fitness landscape adaptation leads to more complicated hypercycle structure (see Fig. 1,2) and allows new behavior, such as autocatalysis.

- Adapted system can be resistant against parasites, even if they were harmful for the classical hypercycle.

- There is a phase transition in the system, which is similar to the error threshold: the mean fitness value and the frequencies distribution in the population tend to stabilize.

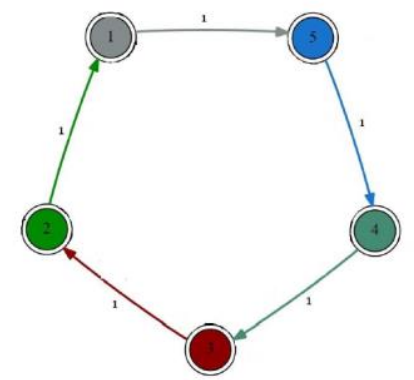

Fig. 1. The original hypercycle.

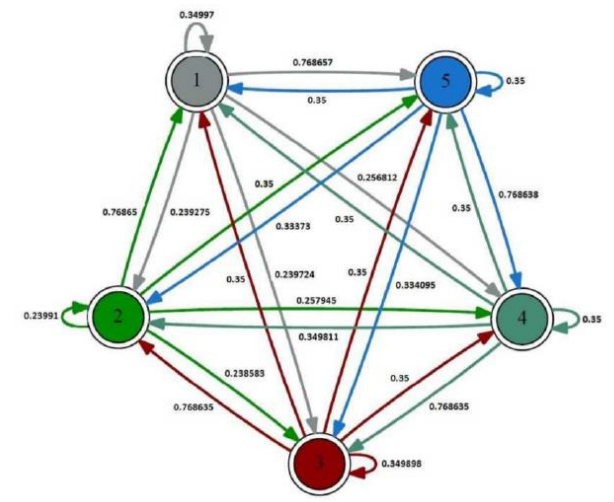

Fig. 2. The adapted hypercycle after 350 iterations of the evolutionary process.

\section{Fitness landscape adaptation process in quasispecies systems}

\subsection{Problem Statement}

Following the classical Crow-Kimura model [10], consider the quasi species system, where each individual is characterized by its genotype of $N$ genes. The distribution of types in the population is defined by the probabilities of getting in one of the $(N+1)$ Hamming classes, where each class is defined by the number of accumulated mutations:

$$
\begin{gathered}
\mathbf{u}(t)=\left(u_{0}(t), \ldots, u_{N}(t)\right) . \\
\dot{\mathbf{u}}(t)=\mathbf{Q}_{m} \mathbf{u}(t)-f(t) \mathbf{u}(t)
\end{gathered}
$$

where the mean fitness is defined by the expression:

$$
f(t)=\sum_{i=0}^{N} m_{i} u_{i}(t),
$$

and $\mathbf{Q}_{m}$ is a fitness matrix, that takes into account transition between classes and mutation rate.

As in the previous section, we suppose that during evolutionary time the system can adapt, which means the variation of the fitness landscape parameters:

$$
\sum_{i=1}^{l} m_{i}(h)=R=\text { const }>0 .
$$

The last expression defines the set $M_{h}$.

In this case, we maximize the mean fitness of the population, varying the parameters of the system under the constraint (11).

\subsection{Optimization process}

Consider the quasispecies system (10) in a steadystate over the set $M_{n}$. Let us denote linear parts of the functions in (10) in a steady state as $\delta \bar{f}(h), \delta \bar{m}, \delta \bar{u}$ correspondingly, varying the parameter $h$ to $h+\Delta h$.

The following holds for the fitness variation:

$$
\delta \mathbf{Q}_{m} \bar{u}+\mathbf{Q}_{m} \delta \bar{u}=\delta \bar{f} \bar{u}+\bar{f} \delta \bar{u},
$$

For the numerical scheme for the extrema search, we can use the combination of linear programming problems:

$$
\left(\delta \mathbf{Q}_{m} \bar{u}, \bar{v}\right) \rightarrow \max ,(\delta m, I=0),-\varepsilon I \leq \delta m \leq \varepsilon .
$$


Here, $\bar{v}$ is the eigenvector in the adjunct eigenvalue problem:

and $\delta m=\delta \mathbf{Q}_{m} \bar{u}$.

$$
\mathbf{Q}_{m}^{T} \bar{v}=\bar{f} \bar{v},
$$

\section{Acknowledgments}

The authors are grateful to A.S. Novozhilov and D. Stepensky for help, useful discussions and comments on the text.

\section{Reference examples}

1. Nowak M.A., Sigmund K. Evolutionary dynamics of biological games. Science. 2004. V. 303. № 5659. P. 793-799.

2. Park S.C., Krug J. Evolution in random fitness landscapes: the infinite sites model. J. Stat. Mech. 2008. V. 4. P. 04014.

3. Yakushkina T., Saakian D.B., Bratus A., Hu C.K. Evolutionary games with randomly changing payoff matrices. J. Phys. Soc. Japan. 2015. V. 84. № 6. P. 064802.

4. Bratus A.S., Drozhzhin S., Yakushkina T. On the Evolution of Hypercycles. Math. Biosci. 2018 (submitted).

5. Bratus A.S., Yakushkina T., Samokhin I. The evolutionary process of fitness landscape adaptation. Math. Biosci. 2018 (submitted).

6. Fisher R.A. The Genetical Theory of Natural Selection. Ed. Bennett. Oxford: Oxford University Press, 1999.

7. Wright S. The roles of mutation, inbreeding, crossbreeding and selection, in evolution. In: Proc. Sixth Int. Congr. Gen. 1932. P. 356-366.

8. Eigen M. Self organization of matter and the evolution of biological macromolecules. Natuwissenschaften. 1971. V. 58. № 10. P. 465523.

9. Eigen M., Schuster P. A principle of natural selforganization. Naturwissenschaften. 1977. V. 64. № 11. P. 541-565.

10. Crow J.F., Kimura M. An introduction to population genetics theory. The Blackburn Press, 1970. 